large cohort of 801 multiple myeloma patients. Haematologica. 2017;102(5):910-921

3. Palumbo A, Anderson K. Multiple myeloma. N Engl J Med. 2011;364:1046-1060

4. Zweegman S, Engelhardt M, Larocca A, EHA SWG on 'Aging and Hematology'. Elderly patients with multiple myeloma: towards a frailty approach? Curr Opin Oncol. 2017;29(5):315-321.

5. O'Donnell EK, Raje NS. Myeloma bone disease: pathogenesis and treatment. Clin Adv Hematol Oncol. 2017;15(4):285-295.

6. Kimura T. Multidisciplinary Approach for Bone Metastasis: A Review. Cancers. 2018;24;10(6).

7. Kyriakou C, Molloy S, Vrionis F, et al. The role of cement augmentation with percutaneous vertebroplasty and balloon kyphoplasty for the treatment of vertebral compression fractures in multiple myeloma: a consensus statement from the International Myeloma Working Group (IMWG). Blood Cancer J. 2019;9(3):27.

8. Terpos E, Ntanasis-Stathopoulos I, Gavriatopoulou M, Dimopoulos MA. Pathogenesis of bone disease in multiple myeloma: from bench to bedside. Blood Cancer J. 2018;8(1):7

9. Engelhardt M, Herget GW, Graziani G, et al. Osteoprotective medication in the era of novel agents: a European perspective on values, risks and future solutions. Haematologica. 2018;103(5):755-758

10. Terpos E, Kleber M, Engelhardt M, et al. European Myeloma Network guidelines for the management of multiple myeloma-related complications. Haematologica. 2015;100(10):1254-1266.

11. Terpos E, Ntanasis-Stathopoulos I, Dimopoulos MA. Myeloma bone disease: from biology findings to treatment approaches. Blood. 2019;133(14):1534-1539.

12. Mhaskar R, Kumar A, Miladinovic B, Djulbegovic B. Bisphosphonates in multiple myeloma: an updated network metaanalysis. Cochrane Database Syst Rev. 2017;12:CD003188.

13. Raje N, Terpos E, Willenbacher W, et al. Denosumab versus zole- dronic acid in bone disease treatment of newly diagnosed multiple myeloma: an international, double-blind, double-dummy, randomised, controlled, phase 3 study. Lancet Oncol. 2018;19(3):370381.

14. Toscani D, Bolzoni M, Ferretti M, Palumbo C, Giuliani N. Role of Osteocytes in Myeloma Bone Disease: Anti-sclerostin Antibody as New Therapeutic Strategy. Front Immunol. 2018;9:2467.

15. Hillengass J, Usmani S, Rajkumar SV, et al. International myeloma working group consensus recommendations on imaging in monoclonal plasma cell disorders. Lancet Oncol. 2019;20(6):e302-e312.

16. Thorsteinsdottir S, Gislason G, Aspelund T, et al. Fractures and survival in multiple myeloma: results from a population-based study. Haematologica. 2019;105(4):1067-1073.

17. Yee AJ, Raje NS. Denosumab for the treatment of bone disease in solid tumors and multiple myeloma. Future Oncol. 2018;14(3):195203.

18. Johnson SK, Knobf MT. Surgical interventions for cancer patients with impending or actual pathologic fractures. Orthop Nurs. 2008:27:160-171.

19. Ward WG, Holsenbeck S, Dorey FJ, Spang J, Howe D. Metastatic disease of the femur: Surgical treatment. Clin Orthop Relat Res. 2003;415:230-244

20. Bryson DJ, Wicks L, Ashford RU. The investigation and management of suspected malignant pathological fractures: a review for the general orthopaedic surgeon. Injury. 2015 Oct;46(10):1891-1899.

21. Engelhardt $M$, Selder $R$, Pandurevic $M$, et al. [Multidisciplinary Tumor Boards: Facts and Satisfaction Analysis of an Indispensable Comprehensive Cancer Center Instrument]. Dtsch Med Wochenschr. 2017;142(9):e51-e60.

22. Sakellariou VI, Mavrogenis AF, Savvidou O, Sim FH, Papagelopoulos PJ. Reconstruction of multiple myeloma lesions around the pelvis and acetabulum. Eur J Orthop Surg Traumatol. 2015;25(4):643-653.

\title{
Animal models of thrombotic thrombocytopenic purpura: the tales from zebrafish
}

\author{
Paul Coppo ${ }^{1}$ and Bernhard Lämmle ${ }^{2,3,4}$
}

${ }^{1}$ Service d'Hématologie, Centre de Référence des Microangiopathies Thrombotiques, AP-HP.6, Paris, France; ${ }^{2}$ Department of Hematology and Central Hematology Laboratory, Inselspital, Bern University Hospital, University of Bern, Bern Switzerland; ${ }^{3}$ Center for Thrombosis and Hemostasis, University Medical Center Mainz, Mainz, Germany and ${ }^{4}$ Haemostasis Research Unit, University College London, London, United Kingdom

E-mail: PAUL COPPO - paul.coppo@aphp.fr or BERNHARD LÄMMLE - Bernhard.laemmle@uni-mainz.de

doi:10.3324/haematol.2019.245043

T hrombotic thrombocytopenic purpura (TTP) results from systemic microvascular von Willebrand factor (VWF)-induced clumping of platelets causing thrombocytopenia, microangiopathic hemolysis, and ischemia in the brain, kidneys, heart, and other organs. ${ }^{1}$ The identification of a severe deficiency of the specific VWF-cleaving protease, now denoted as ADAMTS13, ${ }^{1}$ by Furlan et al. ${ }^{2}$ and Tsai and Lian, ${ }^{3}$ provided an explanation for the accumulation of extremely adhesive, unusually large VWF multimers in the plasma of patients with chronic relapsing TTP first reported by Moake et al. in the 1980s. ${ }^{4,5}$ Severe deficiency of ADAMTS13 activity, caused by biallelic mutations of the encoding ADAMTS13 gene $e^{6.8}$ or, more commonly, by autoantibodies directed against various epitopes of the metalloprotease resulting in functional inhibition of the enzyme and/or the formation of immune complexes and enhanced clearance of ADAMTS13 $2,3,9$ underlies congenital (cTTP) and acquired, autoimmune TTP (iTTP), respectively. ${ }^{10}$

The novel insights into disease mechanisms identified in the laboratory during the past two decades were rapidly and successfully integrated into the clinical management, to the benefit of patients. ${ }^{11}$ Targeted therapies were thereby progressively associated with the historical treatment empirically introduced in the 1970s-1980s, based on repeated plasma exchange, replacement of the deficient protease through fresh-frozen plasma, and corticosteroids, which had already dramatically improved the prognosis of patients with this previously mostly fatal disease. ${ }^{12}$ The successful story of these newly introduced therapeutic approaches include B-cell-depleting monoclonal antibodies that inhibit autoantibody production, ${ }^{13}$ and nanobodies that bind to the VWF A1 domain and inhibit the VWFplatelet glycoprotein Ib interaction. ${ }^{14,15}$ Recently, a recombinant form of human ADAMTS13 was successfully tested in a pharmacokinetics and safety study in 15 patients with cTTP ${ }^{16}$ and is expected to facilitate the management of cTTP and possibly iTTP in the near future. This ongoing development illustrates the strength of translational medicine when basic science and clinical research combine efficiently. This approach allowed TTP to fully enter the exciting era of targeted therapies and personalized medicine.

A crucial advance in TTP pathophysiology was the demonstration of the direct role of ADAMTS13 deficiency 
in the occurrence of the disease, an insight that was strongly supported by the development of animal models of cTTP and iTTP.

\section{Mouse models of thrombotic thrombocytopenic purpura}

Several groups have generated ADAMTS13-deficient $\left(\right.$ ADAMTS $\left.13^{-1}\right)$ mice via gene targeting using a mixedstrain C57BL/6J-129X1/SvJ genetic background. ${ }^{17-19}$ The conclusion from evaluation of these models was that ADAMTS13 is not, per se, sufficient to produce the typical symptoms and signs of TTP in these strains of mice which also had normal survival. ${ }^{17}$ Interestingly, however, when these ADAMTS13\% mice were backcrossed into the CASA/Rk genetic background resulting in substantially higher plasma VWF levels, the resulting ADAMTS13 mice had unusually large VWF multimers, a lower average platelet count and decreased survival, with some mice developing spontaneous TTP manifestations. ${ }^{17}$ Moreover, when these ADAMTS13\% mice of C57BL/6J-129X1/SvJ mixed strain that had been backcrossed into the CASA/Rk background were injected with a strong trigger, i.e. shigatoxin, they developed classic features of acute TTP with severe thrombocytopenia, anemia with schistocytes, increased levels of lactate dehydrogenase (LDH), reflecting both hemolysis and tissue damage, and microthrombi in several organs, especially the heart. ${ }^{17}$ Using very high doses of intravenous recombinant human (rh) VWF (2000 $\mathrm{U} / \mathrm{kg}$ of body weight) as a trigger, even the mixed strain C57BL/6J-129X1/SvJ ADAMTS13\% mice developed acute TTP with severe thrombocytopenia, schistocytic anemia, elevated LDH and myocardial necrosis. ${ }^{19}$ Interestingly, the prophylactic administration of rhADAMTS13 (200 U/kg) protected the mice from acute TTP and even the therapeutic infusion of rhADAMTS13 (320 U/ $/ \mathrm{kg})$, given within 1.5 $\mathrm{h}$ after a challenge with rhVWF, could reduce the severity of TTP findings, demonstrating that this model was useful for investigating the efficacy of prophylaxis and treatment of cTTP. ${ }^{19}$

Thereafter, murine monoclonal antibodies raised in ADAMTS $13 \%$ mice against murine ADAMTS13 were used to generate a mouse model of immune-mediated TTP. ${ }^{20}$ Injecting a combination of two inhibitory monoclonal antibodies into Adamts $13^{+/+}$mice resulted in complete and prolonged inhibition of ADAMTS13 activity for more than 7 days, leading to the appearance of ultralarge VWF multimers in the plasma. In line with the above-mentioned murine cTTP models, TTP-like symptoms in this murine iTTP model did not occur and could only be induced when rhVWF was injected as an additional trigger. $^{20}$

\section{Baboon model of thrombotic thrombocytopenic purpura}

To get closer to man, a non-human primate model of immune-mediated TTP was developed in the baboon (Papio ursinus). ${ }^{21}$ An inhibitory murine anti-human ADAMTS13 monoclonal antibody was administered intravenously to baboons. In contrast to the mouse models of congenital and immune-mediated TTP discussed above, the monoclonal antibody-mediated complete inhibition of ADAMTS13 in the baboon for 4 days resulted in severe thrombocytopenia, microangiopathic hemolytic anemia with increased LDH levels and VWF-rich microthrombi in most organs without the need for an additional trigger. Nevertheless, none of the animals developed severe organ failure or died during the study. ${ }^{21}$ Hence, this model represents early-stage iTTP, and a second hit or prolonged ADAMTS13 inhibition might be needed to induce full-blown TTP with organ failure and death in these baboons.

The baboon iTTP model provided useful data on the feasibility, efficacy and tolerance of new therapeutic strategies for iTTP. ${ }^{22,23}$ Thus, it could be established that injection of a monoclonal antibody blocking the VWF A1 - glycoprotein Ib interaction was a safe and effective means to prevent and treat the early symptoms of iTTP, ${ }^{22}$ whereas the therapeutic application of $\mathrm{N}$-acetylcysteine was unable to resolve established TTP manifestations such as thrombocytopenia, hemolytic anemia and organ damage due to failed resolution of microvascular thrombosis. ${ }^{23}$

These animal TTP models demonstrated that a complete deficiency of ADAMTS13 activity, at least in mice, is not sufficient to produce the typical manifestations of acute TTP which may also depend on the quantity and quality of $\mathrm{VWF}^{24}$ Several authors have postulated that a "second hit" besides severely deficient ADAMTS13 activity may be needed to bring about acute TTP. 25.27 Such second hits triggering the release of VWF from Weibel-Palade bodies in endothelial cells may include extracellular DNA, histones, e.g. resulting from neutrophil extracellular traps, as well as neutrophil peptides 1-3 ( $\alpha$-defensins) or complement activation products such as sC5b-9 which are generated during infections and inflammation. ${ }^{25-27}$ Importantly, however, no direct evidence is available to date to support the causative role of these inflammatory mediators in the pathogenesis of TTP. In this regard, more experimental research using additional models is needed to further unravel the pathophysiology and natural course of TTP and to probe new prophylactic and therapeutic interventions for this rare and severe disease.

\section{What the ADAMTS13\% zebrafish reveals about thrombotic thrombocytopenic purpura}

The zebrafish (Danio rerio) was reported to be a relevant research tool for studying hemostasis and thrombosis, as it displays a high degree of genetic and functional conservation of hemostatic factors including the key functions of VWF, and its nucleated platelets (thrombocytes) seem to have properties comparable to those of human platelets. ${ }^{28}$ The advantages of this vertebrate model include high fecundity, rapid and external embryonic development, and conservation of virtually all hemostatic factors in the zebrafish genome.

In this issue of Haematologica, Zheng et al..$^{29}$ report a new model of cTTP in zebrafish with interesting experiments showing the indispensable role of VWF in the occurrence of TTP and the role of a specific histone as an endothelial activator triggering acute severe disease in the context of severe ADAMTS13 deficiency. The authors took advantage of a double transgenic zebrafish line (gata-1/dsRed and Fli-1/eGFP), which expresses a red fluorescent protein under the gata-1 promoter in erythrocytes and immature thrombocytes and a green fluorescent protein under the fli-1 promoter in the entire vasculature and thrombocytes. 
Using CRISPR/cas9, they created zebrafish lines carrying a null mutation in Adamts13, in VWF, and in both genes. Adamts $13^{-}$zebrafish have higher plasma levels of VWF antigen, larger VWF multimers, and an increased ability of thrombocytes in anticoagulated zebrafish blood to adhere to a fibrillar collagen-coated surface under arterial flow. The Adamts $13 \%$ zebrafish also show an accelerated rate of developing occlusive thrombi in the caudal venules after injury by iron chloride $\left(\mathrm{FeCl}_{3}\right)$, consistent with a prothrombotic phenotype. ${ }^{29}$ Adamts $13 \%$ zebrafish have a reduced number of mature and immature thrombocytes with increased erythrocyte fragmentation, suggesting a mild TTP phenotype in the basal state. The administration of a lysine-rich histone resulted in more severe and persistent thrombocytopenia and a mortality rate of $30 \%$ in ADAMTS $13^{\%}$ versus $10 \%$ in ADAMTS13 $3^{\text {t/ }}$ zebrafish. Both spontaneous and histone-induced TTP phenotypes in Adamts 13 zebrafish were completely prevented when VWF was genetically deleted, proving that the pathophysiological consequence of severe ADAMTS13 deficiency depends entirely on the presence of VWF. On the basis of these results, the zebrafish model developed by Zheng's research team clearly recapitulates the most meaningful features of TTP, which makes this model a promising tool for in-depth exploration of the interrelations between microbes or other environmental influences and the endothelium. Besides a better understanding of the natural history of human TTP, it should also facilitate the assessment of novel therapeutics for TTP and possibly for other arterial thrombotic disorders. One potential limitation of this model is the phylogenetic distance between zebrafish and the human species, raising the question of whether all aspects of hemostasis involving ADAMTS13 will be completely transposable to human physiology and pathology. This new zebrafish TTP model should reveal exciting and still unreported tales of TTP pathophysiology.

\section{References}

1. Kremer Hovinga JA, Coppo P, Lammle B, Moake JL, Miyata T, Vanhoorelbeke K. Thrombotic thrombocytopenic purpura. Nat Rev Dis Primers. 2017;3:17020.

2. Furlan M, Robles R, Galbusera M, et al. von Willebrand factor-cleaving protease in thrombotic thrombocytopenic purpura and the hemolytic-uremic syndrome. N Engl J Med. 1998;339(22):1578-1584.

3. Tsai HM, Lian EC. Antibodies to von Willebrand factor-cleaving protease in acute thrombotic thrombocytopenic purpura. N Engl J Med. 1998;339(22):1585-1594

4. Moake JL, Rudy CK, Troll JH, et al. Unusually large plasma factor VIII:von Willebrand factor multimers in chronic relapsing thrombotic thrombocytopenic purpura. N Engl J Med. 1982;307(23):14321435.

5. Moake JL, Turner NA, Stathopoulos NA, Nolasco LH, Hellums JD. Involvement of large plasma von Willebrand factor (vWF) multimers and unusually large vWF forms derived from endothelial cells in shear stress-induced platelet aggregation. J Clin Invest. 1986;78(6):1456-1461.

6. Levy GG, Nichols WC, Lian EC, et al. Mutations in a member of the ADAMTS gene family cause thrombotic thrombocytopenic purpura. Nature. 2001;413(6855):488-494

7. Joly BS, Boisseau P, Roose E, et al. ADAMTS13 Gene mutations influence ADAMTS13 conformation and disease age-onset in the French cohort of upshaw-Schulman syndrome. Thromb Haemost.
2018;118(11):1902-1917

8. van Dorland HA, Taleghani MM, Sakai K, et al. The International Hereditary Thrombotic Thrombocytopenic Purpura Registry: key findings at enrollment until 2017. Haematologica. 2019;104(10): 2107-2115.

9. Alwan F, Vendramin C, Vanhoorelbeke K, et al. Presenting ADAMTS13 antibody and antigen levels predict prognosis in immune-mediated thrombotic thrombocytopenic purpura. Blood. 2017;130(4):466-471.

10. Scully M, Cataland S, Coppo P, et al. Consensus on the standardization of terminology in thrombotic thrombocytopenic purpura and related thrombotic microangiopathies. J Thromb Haemost. 2017;15(2):312-322.

11. Coppo P, Cuker A, George JN. Thrombotic thrombocytopenic purpura: toward targeted therapy and precision medicine. Res Pract Thromb Haemost. 2018;3(1):26-37.

12. Rock GA, Shumak KH, Buskard NA, et al. Comparison of plasma exchange with plasma infusion in the treatment of thrombotic thrombocytopenic purpura. Canadian Apheresis Study Group. N Engl J Med. 1991:325(6):393-397.

13. Hie M, Gay J, Galicier L, et al. Preemptive rituximab infusions after remission efficiently prevent relapses in acquired thrombotic thrombocytopenic purpura. Blood. 2014;124(2):204-210.

14. Peyvandi F, Scully M, Kremer Hovinga JA, et al. Caplacizumab for acquired thrombotic thrombocytopenic purpura. $N$ Engl J Med. 2016;374(6):511-522

15. Scully M, Cataland SR, Peyvandi F, et al. Caplacizumab treatment for acquired thrombotic thrombocytopenic purpura. $N$ Engl J Med. 2019;380(4):335-346

16. Scully M, Knobl P, Kentouche K, et al. Recombinant ADAMTS-13: first-in-human pharmacokinetics and safety in congenital thrombotic thrombocytopenic purpura. Blood. 2017;130(19):2055-2063.

17. Motto DG, Chauhan AK, Zhu G, et al. Shigatoxin triggers thrombotic thrombocytopenic purpura in genetically susceptible ADAMTS13-deficient mice. J Clin Invest. 2005;115(10):2752-2761

18. Banno F, Kokame K, Okuda T, et al. Complete deficiency in ADAMTS13 is prothrombotic, but it alone is not sufficient to cause thrombotic thrombocytopenic purpura. Blood. 2006;107(8):31613166.

19. Schiviz A, Wuersch K, Piskernik C, et al. A new mouse model mimicking thrombotic thrombocytopenic purpura: correction of symptoms by recombinant human ADAMTS13. Blood. 2012;119(25): 6128-6135.

20. Deforche L, Tersteeg C, Roose E, et al. Generation of anti-murine ADAMTS13 antibodies and their application in a mouse model for acquired thrombotic thrombocytopenic purpura. PLoS One. 2016;11(8):e0160388.

21. Feys HB, Roodt J, Vandeputte N, et al. Thrombotic thrombocytopenic purpura directly linked with ADAMTS13 inhibition in the baboon (Papio ursinus). Blood. 2010;116(12):2005-2010.

22. Feys HB, Roodt J, Vandeputte N, et al. Inhibition of von Willebrand factor-platelet glycoprotein $\mathrm{Ib}$ interaction prevents and reverses symptoms of acute acquired thrombotic thrombocytopenic purpura in baboons. Blood. 2012;120(17):3611-3614.

23. Tersteeg C, Roodt J, Van Rensburg WJ, et al. N-acetylcysteine in preclinical mouse and baboon models of thrombotic thrombocytopenic purpura. Blood. 2017;129(8):1030-1038.

24. Vanhoorelbeke K, De Meyer SF. Animal models for thrombotic thrombocytopenic purpura. J Thromb Haemost. 2013;11 (Suppl 1):210

25. Fuchs TA, Kremer Hovinga JA, Schatzberg D, Wagner DD, Lammle B. Circulating DNA and myeloperoxidase indicate disease activity in patients with thrombotic microangiopathies. Blood. 2012;120(6): 1157-1164.

26. Miyata T, Fan X. A second hit for TMA. Blood. 2012;120(6):11521154.

27. Staley EM, Cao W, Pham HP, et al. Clinical factors and biomarkers predict outcome in patients with immune-mediated thrombotic thrombocytopenic purpura. Haematologica. 2019;104(1):166-175.

28. Weyand AC, Shavit JA. Zebrafish as a model system for the study of hemostasis and thrombosis. Curr Opin Hematol. 2014;21(5):418422.

29. Zheng L, Abdelgawwad MS, Zhang D, et al. Histone-induced thrombotic thrombocytopenic purpura in adamts13-/- zebrafish depends on von Willebrand factor. Haematologica. 2020;105105(4):11071119 . 